\title{
Prevalence of Gingival Recession among the Different Races of Patients Reporting to Penang International Dental College
}

\author{
J. Sabarinathan ${ }^{1}$, M.N. Prabhu ${ }^{2}$, Lo Tze Lui ${ }^{3}$, Vanessa Chung ${ }^{3}$, Tan Shiau Lin ${ }^{3}$, Vivien Chew ${ }^{3}$, Kho Aik Jin ${ }^{3}$ \\ ${ }^{1}$ Department of Orthodontics, Penang International Dental College, Penang, Malaysia \\ ${ }^{2}$ Department of Periodontics, MFDS RCPS(GLASGOW), MFGDP(UK) RCS Eng \\ ${ }^{3}$ Penang International Dental College, Penang, Malaysia
}

Abstract Gingival recession is one of the most prevalent problems seen among the patients visiting to our dental hospital. Recession is caused by many factors including plaque, faulty brushing habits and trauma from occlusion. The prevalence of recession among the various races of people visiting our hospital is estimated in this study.

Keywords: Gingiva, faulty brushing, mucogingival surgery, prevalence, plaque

Cite This Article: J. Sabarinathan, M.N. Prabhu, Lo Tze Lui, Vanessa Chung, Tan Shiau Lin, Vivien Chew, and Kho Aik Jin, "Prevalence of Gingival Recession among the Different Races of Patients Reporting to Penang International Dental College.” International Journal of Dental Sciences and Research, vol. 2, no. 4A (2014): 1-3. doi: 10.12691/ijdsr-2-4A-1.

\section{Introduction}

This article discusses the prevalence of gingival recession among the different races of patients reporting to Penang International Dental College (PIDC). Gingival recession is defined as the exposure of the root surface by an apical shift in the position of the gingiva. There are 2 types of recession, i.e. visible which is clinically observable and hidden, which is covered by gingiva and can only be measured with probe. Gingival recession may be localized or generalized. Position of the gingiva can be actual or apparent. Actual position is the level of epithelial attachment on the tooth, i.e. from the cementoenamel junction to the probable depth of the pocket, whereas apparent position is the level of crest of the gingival margin, i.e. from the cementoenamel junction to the gingival margin [1]. Plaqueinduced gingival inflammation is the primary etiological factor responsible for gingival recession; next most common cause is faulty tooth brushing. Other secondary/contributing factors of gingival recession are broadly categorized as: anatomical, habits, iatrogenic factors and physiologic factors. Anatomical factors include tooth malposition or position of the tooth in the arch, presence of dehiscence and fenestrations, gingival ablation from soft tissues like cheek, lips, etc, root-bone angle and mesiodistal curvature of the tooth surface. Habits include faulty tooth brushing or brushing with hard bristles may lead to gingival recession [2]. Iatrogenic factors include primary trauma from occlusion and improper restorations. Physiological factors include aging. The clinical significance of gingival recession are exposed root surface which may be extremely sensitive, hyperemia of the pulp may result due to gingival recession, interproximal recession creates oral hygiene problems resulting in plaque accumulation [3].

\section{Aims}

The aim is to estimate gingival recession in patients of different races coming to PIDC. The races in consideration are - Malay, Chinese and Indian.

\section{Materials and Methods}

(a) Instruments used to determine gingival recession were: periodontal probe, mouth mirror, explorer

(b) The data was collected based on the observation made on the patient. Case sheet proforma was used to collect their information. Exclusion criteria was done for patients with systemic diseases, patients who had undergone any type of periodontal management in the past 6 months and also smokers.

(c) Classification of gingival recession according to PD Miller's:

Class I- marginal tissue recession that does not extend to the mucogingival junction. There is no loss of bone or soft tissue in the interdental area. This can be narrow or wide.

Class II - marginaltissue recession extends to or beyond the mucogingival junction. There is no loss of bone/soft tissue in the interdental area. This typeof recession can be subclassified into wide and narrow.

Class III - Marginal tissue recession extends to or beyond the mucogingivaljunction. There is bone and soft tissue loss interdentally or malpositioning of the tooth.

Class IV - Marginal tissue recession extends to or beyond the mucogingivaljunction. There is severe bone and soft tissue loss interdentally or severe tooth malposition [4]. 


\section{Results}

Data were collected from 100 patients who had sought consultation at the department of Periodontics, according to their races and gender; 52 males and 48 females were enrolled in the study.

Out of 100 patients, 62 patients were found to have gingival recession, 32 were male and 30 were female.

Based on this study it is very clear that class I recession is more common among Chinese females and is least found in Malay males.

Based on this study it is clear that class II recession is more common among Chinese females and is least found in Malay females.
Based on this study it is very clear that class III recession is more common among Chinese and Indian males and is least found in Malay females.

Based on this study it is very clear that class IV recession is more common among Indian males and females and is least found in Malay and Chinese.

4.1. Table 1

\begin{tabular}{|c|c|c|c|}
\hline $\begin{array}{c}\text { RECESSION } \\
\text { TYPE }\end{array}$ & $\begin{array}{c}\text { MALAY } \\
\text { M / F } \%\end{array}$ & $\begin{array}{c}\text { CHINESE } \\
\text { M / F } \%\end{array}$ & $\begin{array}{c}\text { INDIAN } \\
\text { M / F } \%\end{array}$ \\
\hline CLASS I & $11 / 18.7$ & $16.8 / 24$ & $18 / 13.5$ \\
\hline CLASS II & $1.6 / 0$ & $0.5 / 2.1$ & $2.1 / 1.9$ \\
\hline CLASS III & $0.4 / 0$ & $1.8 / 0.3$ & $0.2 / 1.8$ \\
\hline CLASS IV & NIL & NIL & $0.2 / 0.2$ \\
\hline
\end{tabular}

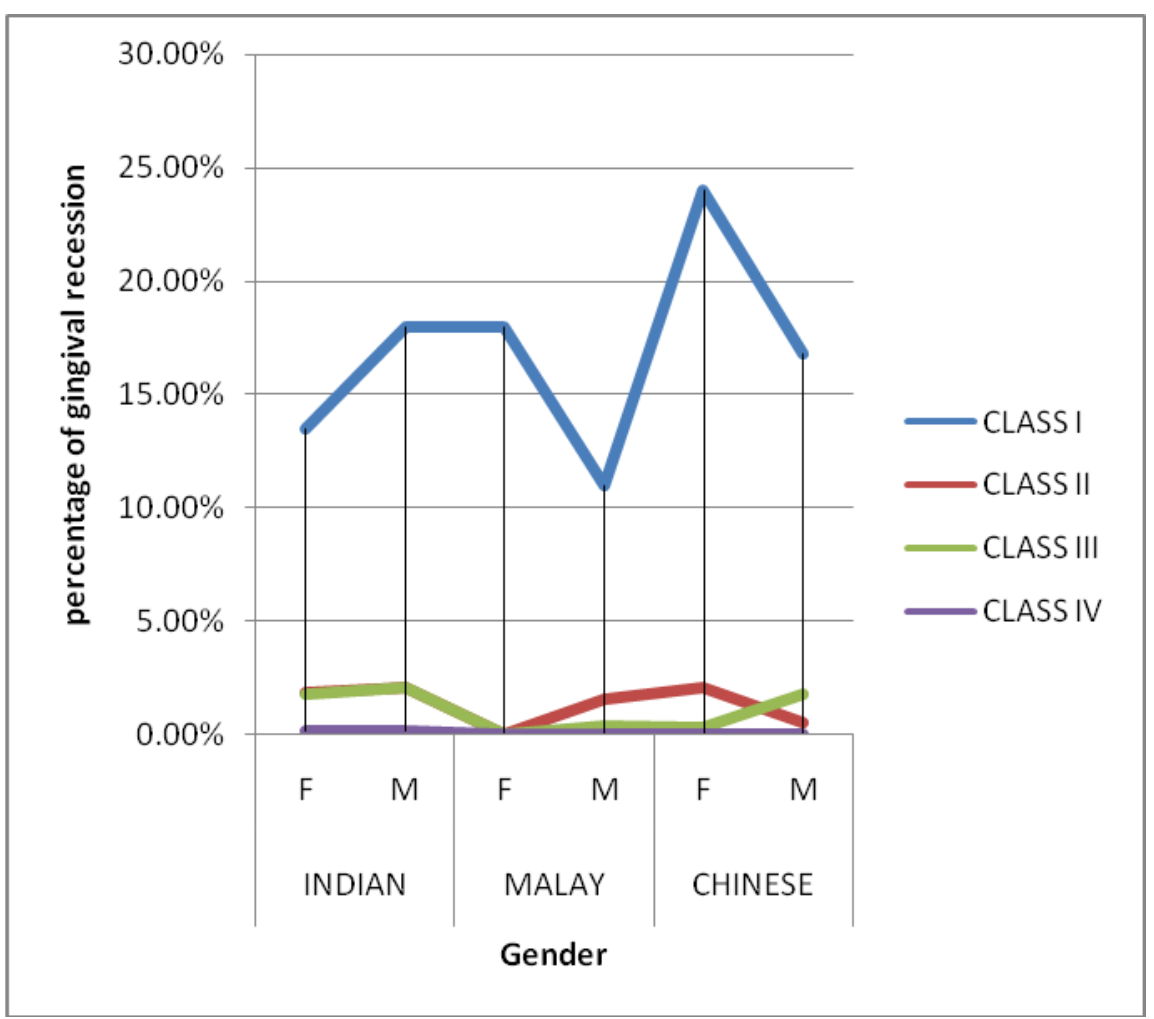

\section{Discussion}

By clinical definition, recession is exposure of the root surface by an apical shift in the position of the gingiva. Actual position is the level of the coronal end of the epithelial attachment on the tooth, whereas the apparent position is the level of the crest of the gingival margin. Severity of recession is determined by the actual position of the gingiva, not its apparent position [1].

The aim is to estimate gingival recession in patients of different races coming to Penang International Dental College. The races in consideration are - Malay, Chinese and Indian.

Based on the above results it is clear that the Chinese females are more prone to the most common type of recession- the class I recession.

According to the results of the present study, gingival recession was the result of more than one factor acting together. Horizontal brushing method, usage of medium type toothbrush, poor oral hygiene, food impaction and smoking were found to be more associated with gingival recession. This is in correlation with the studies done earlier [5].
Proper management of recession restores back the gingiva to health. Various surgical options are available to manage recession. Mucogingival surgery is one of the methods to treat gingival recession. It consists of "plastic surgical procedures for the correction of gingiva, mucous membrane relationships that complicate periodontal diseases and may interfere with the success of periodontal treatment." The objectives of mucogingival surgery are widening the zone of attached gingiva, coverage of denuded roots, removal of aberrant frenum, creation of some vestibular depth when it is lacking and as an adjuvant to routine pocket elimination procedures. The surgical design is based on the type of recession [6].

\section{Conclusion}

This study gives an idea about the race which is more prone to recession. Also it highlights the most common type of recession associated with the race. The etiology behind the recession is also partly explained. It is always better to treat recession at the earlier stages [7]. Various surgical and non surgical options are available for the same. The dentists should also discuss and explain to the 
patients about the proper brushing techniques, as this will go a long way in preventing recession [8].

\section{References}

[1] Ajay et al, "Effective management of gingival recession defects using periosteal pedicle grafts”, e-Journal of Dentistry, Vol 2(3), 193-199, July - Sep 2012.

[2] Moawia et al, "The etiology and prevalence of gingival recession”, The Journal of the American Dental Association, 134, 220-225, February 2003.

[3] Kaumudi.et al, “Gingival Recession: Intra-Oral Distribution and Associated Factors”, Journal of Periodontology, Vol. 65(9), 86487, September 1994.
[4] Newman, Takei, et al, Clinical Periodontology, Saunders, Missouri, 2006, 1014-1015.

[5] Ahmed Khochtet al, "Gingival Recession in Relation to History of Hard Toothbrush Use”, Journal of Periodontology, Vol. 64(9), 900-905, September 1993.

[6] Thomas W. Oates, et al. "Surgical Therapies for the Treatment of Gingival Recession. A Systematic Review”, Annals of Periodontology, Vol. 8(1), 303-320, December 2003.

[7] G. Zucchelli, et al, "Clinical and Anatomical Factors Limiting Treatment Outcomes of Gingival Recession: A New Method to Predetermine the Line of Root Coverage", Journal of Periodontology, Vol. 77(4), 714-721, April 2006.

[8] C. Ganss, et al, "Tooth brushing habits in uninstructed adults-frequency, technique, duration and force”, Clinical Oral Investigations, Vol13( 2), 203-208,June 2009. 